\title{
Ärztlicher Notfalldienst: Qualitätskriterien
}

Sehr geehrter Herr Präsident, sehr geehrte Damen und Herren

Im Auftrag des Vorstandes sowie der kantonalen Bezirkspräsidenten des Aargauischen Ärzteverbandes gelangen wir in folgender Angelegenheit an Sie: Ihr Vorstandsmitglied Dr. Reto Laetsch publizierte in der Schweizerischen Ärztezeitung (2001;82[12]:586-7) im Namen des Forums Notfallmedizin die Qualitätskriterien für den ärztlichen Notfalldienst. Wie seinem Schreiben vom 13. April $2001 \mathrm{zu}$ entnehmen ist, haben wir von ihm die Präzisierung dieser Darlegung erhalten - wie er sie auch anlässlich der Ärztekammer am 5. Mai 2001 erläutert hat - mit der Bemerkung, dass es sich dabei um fakultative Empfehlungen handelt. Da sein Artikel unter der Rubrik "Politik, Ökonomie und Recht» ohne Hinweis auf einen Empfehlungscharakter erschien, hat dieser für den unvoreingenommenen Leser Richtliniencharakter. Wir erlauben uns deshalb nochmals, auf die offensichtlich ungenügend bedachten Konsequenzen eines derartigen Vorgehens hinzuweisen.

Im aargauischen Gesundheitsgesetz wird festgehalten, dass alle freipraktizierenden Ärzte am Notfalldienst in angepasster Weise teilnehmen. Der Aargauische Ärzteverband hat aufgrund dieser Gesetzesvorlage ein Reglement erarbeitet, welches den Bezirksverbänden weitgehende Freiheiten in der Organisation des Notfalldienstes gibt, wobei in unseren ländlichen bis kleinstädtlichen Bezirken schwergewichtig alle freipraktizierenden Ärzte am Notfalldienst partizipieren.

Bei Umsetzung der Qualitätskriterien würde kaum noch die Hälfte der Ärzte am Notfalldienst teilnehmen, wobei davon ausgegangen werden muss, dass auch seit längerer Zeit tätige Grundversorger nicht gewillt sein dürften, zusätzliche finanzielle und zeitliche Investitionen zur Ausübung des Notfalldienstes zu tätigen. Dies vor allem auch unter dem Aspekt, dass eigentliche Notfälle mit Reanimationssituatio- nen im Notfalldienst sehr selten auftreten und es sich bei den meisten Einsätzen um Notfälle aus Sicht der Patienten handelt, wo neben dem allgemeinärztlichen Wissen vor allem der gesunde Menschenverstand gefragt ist. Im Kanton werden - teilweise organisiert durch den Ärzteverband - in regelmässigen Abständen auch weiterhin Fortbildungsveranstaltungen in Notfallmedizin angeboten, deren Besuch allgemein empfohlen wird, welcher aber nicht Voraussetzung für die Teilnahme am Notfalldienst war.

Sollte der FMH-Zentralvorstand die Qualitätskriterien für eine Teilnahme am Notfalldienst in der erwähnten Form sanktionieren, würde dies die Organisation eines geordneten Notfalldienstes im Kanton erschweren bis verunmöglichen und eine echte Konfliktsituation auch mit den Gesundheitsbehörden respektive dem Gesundheitsgesetz schaffen, welche in keiner Relation zum Nutzen für die Bevölkerung steht, wenn tatsächlich alle am Notfalldienst teilnehmenden Ärzte den Advanced Life Support (ALS) garantieren könnten. Alternativ, jedoch folgerichtig müsste in allen Curricula diese Ausbildung integriert werden, da jederzeit bei allen Fachgruppen, welche im Minimum Lokalanästhetika verwenden, lebensbedrohliche Notfallsituationen auftreten können. Falls ALS für den Notfalldienst unabdingbar sein sollte, dann müssten alle Ärzte dazu befähigt sein.

Weiter stellt sich uns die Frage der rechtlichen Konsequenzen, wenn z.B. ein Arzt im Notfalldienst zu einer Laienreanimation gerufen wird und keinen Defibrillator bei sich hat. Für einen Juristen wäre es ein leichtes, sich auf die Qualitätskriterien abzustützen, vor allem auch, wenn das notwendige Instrumentarium wie angekündigt zusätzlich publiziert wird.

Sollten die Qualitätskriterien, welche für alle Ärzte verbindlich sind, nicht durch die standeseigenen dafür vorgesehenen (demokratischen) Strukturen - sprich Kommission für Qualitätssicherung - abschliessend beurteilt werden? Ein entsprechender Antrag wird vom Aargauischen Ärzteverband an der nächsten Ärztekammer vorgelegt.

Gerne erwarten wir Ihre Stellungnahme und beantragen, diesen Brief sowie Ihre Antwort in der Schweizerischen Ärztezeitung zu publizieren.

Mit freundlichen Grüssen

Dr. Andreas Haefeli, Präsident

Dr. Rudolf Stäubli, Vizepräsident 


\section{Replik}

Sehr geehrter Herr Präsident, sehr geehrte Damen und Herren

Für Ihr Schreiben vom 31. Mai 2001 danken wir Ihnen und nehmen dazu gerne Stellung.

Die Plattform Schweizerisches Rettungswesen der FMH ist gewissermassen der «think tank» der FMH für die notfall- und rettungsmedizinischen Belange, insbesondere auf dem Gebiet der ärztlichen Aus-, Weiter- und Fortbildung. Sie besteht aus Vertretern der SGAM, SGAR, SGC, SGIM, SGI, SGP, SGNOR, des IVR, des Gst UG San sowie des SRK unter dem Vorsitz des ZV-Mitglieds Dr. R. Laetsch.

Dass die Publikation der Qualitätskriterien für den ärztlichen Notfalldienst in der Rubrik «Politik, Ökonomie und Recht» der Schweizerischen Ärztezeitung erfolgte und damit offenbar den Anstrich einer amtlichen Verlautbarung bekommen hat, war alles andere als beabsichtigt.

Es ist hier nochmals zu betonen, dass die Qualitätskriterien für den ärztlichen Notfalldienst eine proaktive Expertenempfehlung zur zukünftigen qualitativen Gestaltung des ärztlichen Notfalldienstes sind und mit Bedacht also solche - und nicht etwa als Richtlinien - bezeichnet worden sind.

Die Plattform liess sich bei der Formulierung der Qualitätskriterien für den ärztlichen Notfalldienst u. a. von folgenden Überlegungen leiten:

Der ärztliche Notfalldienst ist ein Teil der Grundversorgung und soll daher wo immer möglich, und um den heutigen Publikumsansprüchen gerecht zu werden, von Trägern eines Grundversorgertitels mit entsprechender Tätigkeit geleistet werden. Damit ist etwas präziser ausgeführt, was Sie in Ihrem Schreiben «allgemeinärztliches Wissen plus gesunder Menschenverstand" als qualitatives Erfordernis nennen.

Die Dienstärztinnen und Dienstärzte sollen sich die Kompetenzen für ihre Aufgabe, in lebensbedrohlichen Situationen bis zum Eintreffen der spezialisierten Rettung wirkungsvolle Hilfe leisten zu können, in strukturierter Form erwerben können. Die SGAM hat den entsprechenden Kurs bereits in ihr Ausbildungsprogramm aufgenommen. Mit dem Kriterium "Repetitionskurs" will die Plattform auf die Bedeutung des notfallmedizinischen Trainings ge- rade wegen der üblicherweise nicht besonders häufig vorkommenden Reanimationssituation im ärztlichen Notfalldienst hinweisen: Fitness für die Aufgabe im Rahmen der kontinuierlichen beruflichen Entwicklung.

Die Überlegungen der Plattform zur Qualifikation der Dienstärztinnen und Dienstärzte haben bereits dazu geführt, dass die notfallmedizinischen Kurse der SGAM, der SGNOR und der Armee nun gegenseitig anerkannt werden. Weitere Bestrebungen, das Kursangebot zu verbessern, sind in vollem Gange.

Im Rahmen der Ausrüstungsempfehlungen stellt die Verfügbarkeit über einen Defibrillator ein grösseres Problem dar, einer der Gründe dafür, dass die Ausrüstungsliste noch nicht verabschiedet werden konnte. Auf der einen Seite stellt die Frühdefibrillation unbestrittenermassen die wirksamste Massnahme beim Patienten mit Kreislaufstillstand dar, eine Erkenntnis, die sich die Laienhelfer bereits zu eigen gemacht haben und daran sind, sie praktisch umzusetzen. Auf der anderen Seite kann die Anschaffung eines halbautomatischen oder automatischen Defibrillators zu den heutigen Bedingungen dem Dienstarzt nicht zugemutet werden, und die gemeinsame Nutzung, z.B. innerhalb eines Dienstarztkreises, dürfte meist an organisatorischen Schwierigkeiten scheitern. Leider waren bisher alle Bemühungen, Defibrillatoren für Arztpraxen erschwinglich zu machen, erfolglos. Diese Voraussetzungen erschweren eine angemessene Formulierung in der Ausrüstungsliste, die nicht die Gefahr von Haftpflichtansprüchen in sich birgt. Hier wären wir auch Ihnen sehr dankbar um Ihren Beitrag an die Problemlösung.

Ihrer Frage, ob die Qualitätskriterien für den ärztlichen Notfalldienst durch die Kommission für Qualitätssicherung abschliessend beurteilt werden sollten, stehen wir durchaus positiv gegenüber.

Wir hoffen, Ihnen mit dieser Stellungnahme, die wir Ihrem Wunsch entsprechend zusammen mit Ihrem Schreiben vom 31. Mai 2001 in der Schweizerischen Ärztezeitung publizieren werden, zu dienen und verbleiben

mit freundlichen, kollegialen Grüssen

$\mathrm{F} \mathrm{M} \mathrm{H}$

Dr. med. H. H. Brunner, Präsident

Dr. med. R. Laetsch, Mitglied Zentralvorstand 